\title{
Wireless technologies for Controlling a Traffic Lights Prototype
}

\author{
J.P.P. Cunha \\ Mechanical Engineering MSc \\ Instituto Superior Técnico, Universidade de Lisboa \\ Lisbon, Portugal \\ joao.p.cunha@tecnico.ulisboa.pt
}

\author{
C. Cardeira, N.C. Batista and R. Melício \\ IDMEC, Instituto Superior Técnico, Universidade de Lisboa \\ Departamento de Física, Escola de Ciências e Tecnologia, \\ Universidade de Évora \\ Portugal
}

\begin{abstract}
bstract - This paper presents a traffic light control system based on wireless communication technologies. Traffic density is increasing at an alarming rate in developing countries which calls for intelligent dynamic traffic light control systems to replace the conventional manual and time based ones. The approach followed in this paper is based in a secure wireless sensor network to feed real time data to the intelligent traffic light control. A physical prototype was implemented for experimental validation. The physical prototype showed robustness against local failures or unforeseen cases showing that the communication between modules keeps an acceptable packets received ratio.
\end{abstract}

Keywords-metropolitan area networks; communication system traffic control; electronic circuits; peer-to-peer computing; networked control systems.

\section{INTRODUCTION}

Every major city has a large part of the population travelling by car or by public transportation, and traffic jams are a frequent problem [1]. Better public transportation is the sustainable long-term solution to road traffic jams but a nearer term solution is also required. The most obvious solution is to build new roads but, in urban areas, this is generally not feasible, due to a lack of suitable land or insufficient funds [2]. Hence, the traffic density is increasing at an alarming rate in developing countries which calls for intelligent traffic lights control to replace the conventional manual and time based ones [8]. To solve this problem in Lisbon, Portugal, since 1985, adopted a system called Gestion Electronique de Règulation en Temps Réel pour l'Urbanisme (GERTRUDE) [3].

The GERTRUDE system is a centralized management traffic control system that, from data supplied by sensors deployed in the pavement $(1400+)$ and connected to a road control central, allows the comparison between traffic flows and react in real time to each situation through traffic lights [4]. Currently, GERTRUDE is a relatively old system. Even though the algorithm is still relevant and fully adequate, the hardware can be improved. Today, ways of thinking the cities are different. The quality of life in the cities is now the main goal of the cities' strategic planning.

New traffic systems have been developed to provide answers to these strategies [5]. The new traffic system areas, namely, the intelligent transportation system (ITS), combine the existing technology with artificial intelligence and wireless communication technologies (WCT) that, in the limit, would truly think for themselves [6].

An ITS has to capture many environmental measurements in order to improve traffic. Using suitable protocols, WCT are also able to acquire real-time data in a reliable way. Accurate and reliable real-time traffic data monitoring systems are essential for the efficient and successful execution of all ITS systems [7,8]. For example, a traffic lights network of a whole city can use weather and climate (air pollution) values to choose sequences of time to alternate traffic lights, in addition to the collected real-time data concerning vehicles (speed or queue length) $[9,10]$.

The strategic works that have been carried out belong to a wider field of cities management, the so called Smart Cities. Smart Cities go far beyond traffic management to include energy, water, and waste management among many other managing aspects of the Cities. However the use of WCT in cities remains relatively limited. Regarding traffic control, solutions exist for connecting traffic lights using the Global System for Mobile Communications (GSM) network operators. They rely on an unique GSM operator and, at the local level, some solutions exist for acquiring data from sensors (bellow the pavement) and wirelessly transmit their information to the local controller [11].

The WCT have been developed but one in particular has received a lot of attention: ZigBee. This is a low power wireless networking standard designed for controlling and monitoring applications. The ZigBee standard was prepared by an industry consortium: the ZigBee alliance [12].

Using WCT, it becomes easier adapt to fluctuations, as the number and type of sensors may be largely increased. Wireless sensors can be installed almost everywhere and provide important additional information to optimize traffic management.

In local area solutions, data acquired in a certain location is used there to know what road to open, decided by any preprogrammed or pre-defined algorithm or local sensors. In wider area solutions for traffic control, the system processes the data acquired in the various locations with devices capable of doing so. They send information to all the local controllers not only to improve the traffic flow in that intersection but also in the neighbor intersections. This type of system also allows tracking of priority vehicles, like ambulances, to accommodate easier and quicker transportation of critical 
patients and public transportation priority roads. However, there are some small issues with the system as well, such as problems with communication between controllers. The reaction capability of the system is limited to the central data analysis and there are no proactive capabilities aiming to prevent possible and, eventually, foreseeable events [13].

A functioning and applicable solution to a traffic control system has to be robust. The malfunction of any modules at any time is a possibility, so the choice of the communications topology has be done to effectively deal with this issues. Point-to-point and Star-type networks were ruled out because solutions based on networks in which the communications in the grid depend from the functioning of one module have to be avoided. A mesh-like grid is a type of network that is not dependent from only one point at any time. Moreover, several paths are available for the same communication, which allows the prototype to carry its purpose successfully even if there are missing or malfunctioning modules.

This main contribution of this paper is the definition and performance analysis of a reliable communications infrastructure to maintain and continuously refresh the real time database of sensors and actuators that support the optimization methods for the intelligent traffic management, and ascertain that the existing traffic control systems can be improved by the use of wireless technologies [14]. Wireless technologies present advantages and drawbacks but the tradeoff between them seems in favor of their use for traffic sensing and control.

\section{PROTOTYPE}

\section{A. Configuration}

For the intelligent traffic lights control, the type of the communications grid used is vital for the success of the system. The communication grid is composed by several nodes, arranged in a grid that can communicate with each other allowing messages to flow from any module to another throughout the others.

The configuration of the traffic lights prototype can be extrapolated to a real case. The prototype consists in one microcontroller Arduino per module that controls four traffic lights with three lights each, with the standard colors: red, yellow and green.

The chosen protocol was ZigBee. ZigBee is a reliable, low power, low cost and efficient technology for the creation of information networks; a specification of high level communication protocol based on the LR-WPAN IEEE 802.15.4 standard extending that definition by developing the higher layers of the standard. The ZigBee modules used in the field tests were built taking in consideration the most desirable features of the ZigBee protocol: the ability to create the data network mesh, the self-healing and security [15,16-18]. The ZigBee mesh with all the possible modules: the coordinator, the router and the end-points are shown in Fig. 1.

The Application Programming Interface (API) is built on a frame based protocol consisting of five main parts: i) the start delimiter which is always " $0 \times 72$ "; ii) the length of frame data that has a two-byte value specifying the number of bytes that will be contained in the frame data field, not including the checksum; iii) the frame data portion that is composed by the API identifier and specific data; iv) the contents of the specific data that will change according to the API identifier; and finally, v) the checksum that is a byte containing the hash sum of the API frame bytes.

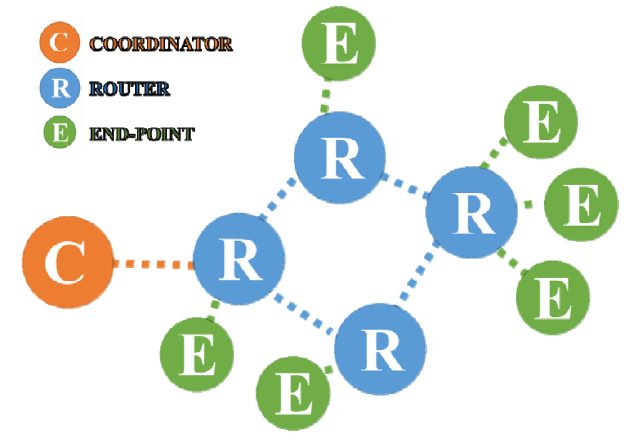

Fig. 1. ZigBee mesh with all the possible modules.

\section{B. Protocol Developed}

The communication protocol is one of most sensible aspects about any communication system, not to mention the safety and privacy. At this phase of building the prototype it was important to focus not only on the communication and message delivery in the system but also receiving feedback from each module, to acknowledge the success of the transmission. An encrypted password is used to protect any relevant information $[13,19]$.

The protocol to communicate between every pair of modules in the grid was hence developed. At this phase no special protocol message size optimization was carried out to keep the messages readiness. The validation of liveness and absence of deadlocks will be needed in a more complete version of the protocol developed. The hash symbol was used as a general separator, and SMSTART as a message starter. The final protocol format is given by:

\section{STARTER\#INTERSECTION\#TRAFFICLIGHT\# ... ... OPERATION MODE\#ORDER\#PASSWORD\#CRC}

If the coordinator needed to send a specific red light manual order to the third traffic light in the intersection number 23 , the message sent, according to this protocol, given by:

\section{SMSTART\#023\#3\#MANUAL\#RED\#PASSWORD\#CRC}

If the coordinator needed the intersection to start the selfmanagement automatic mode the message is given by:

\section{SMSTART\#023\#ALL\#AUTO\#ON\#PASSWORD\#CRC}

The coordinator sends the information to different modules through a broadcast message. Each module interprets the message and checks if the message is assigned to it or not. This was the implementation for the prototype. A real wider 


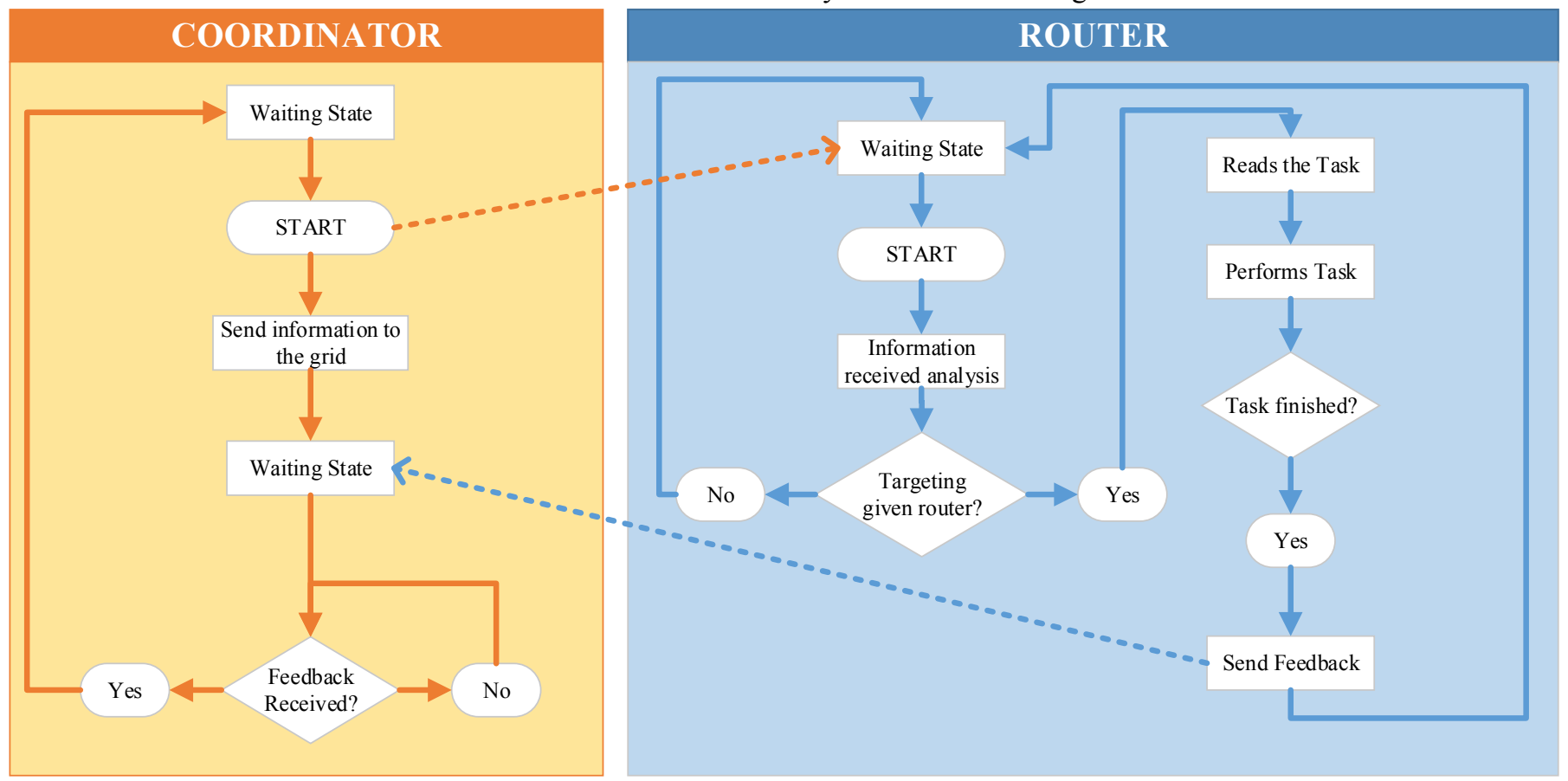

Fig. 2. Flowchart of the System.

\section{EXPERIMENTAL SETUP}

To develop this work 2 Arduinos and 3 ZigBee modules were used. With these, a network between 2 routers (in Attention Command Mode (AT)) and a coordinator (in API mode) was formed.

The AT mode is a more easy and "transparent" mode comparing with API mode. In this mode, any data sent to the XBee module is immediately sent to the correct remote module using the Destination Address. There is no need packet formation, only a simple two way Serial data transmission between two modules. The API mode requires the formation of packets. All data must be formatted in frames with Destination Information and payload. In this mode is possible to assess the general status and quality of the mesh created.

With the coordinator constantly connected to a computer receiving and sending information. Using the X-CTU, the property program of the company that develops ZigBee, it was possible to access the list of packets received on the coordinator. A testing program was loaded into each of the routers for them to send information to the coordinator that could be worked on. The test program consisted in increments of a number every $5 \mathrm{~s}$, information which was sent from ZigBee by each of the routers on broadcast to the coordinator.

These tests were made to verify the communication limits between the various modules. The tests were carried out in the Control Automation and Robotics Laboratory of the Center of Intelligent Systems of IDMEC/Instituto Superior Técnico.
With the list of packets received created, a MATLAB script was developed to treat and analyze this data. Both the packets received and those not received were registered in order to be later analyzed for this study.

For prototype construction, the structure of a few traffic lights was modeled in a CAD program and then printed on a 3D printer, as shown in Fig. 3.

For electronics and connections some LED strips of various colors (Red, Yellow and Green) were prepared. Using transistors to help in the commuting of the traffic lights a circuit board was created to connect the traffic lights to Arduino in order for them to be controlled.

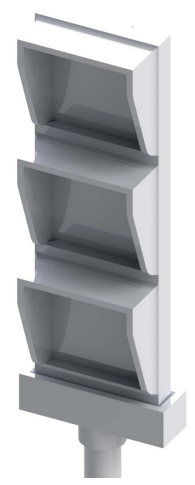

Fig. 3. 3D Model of the Prototype.

The testing program was replaced by one that would interpret the developed protocol presented earlier. All the tests to assure the information sent by the coordinator and vice-versa were interpreted correctly were made. 


\section{EXPERIMENTAL RESULTS}

As a simulation of possible range or connection related issues five tests were done in five different situations. Measurements were taken in the same place and same distances. The following cases were considered to check the Packet Reception Ratio (PRR):

Case 1: All the modules within the communications range, as shown in Fig. 4; Case 2: One module disconnected. The other is located in the limit range, as shown in Fig. 5; Case 3: All modules connected but one in the limit range, as shown in Fig. 6; Case 4: One module out of the range of the coordinator but in the range of other modules, as shown in Fig. 7; Case 5: Same as Case 4, all devices kept the same position except the device closer to the coordinator that is moved to the limit range of the coordinator, as shown in Fig. 8.

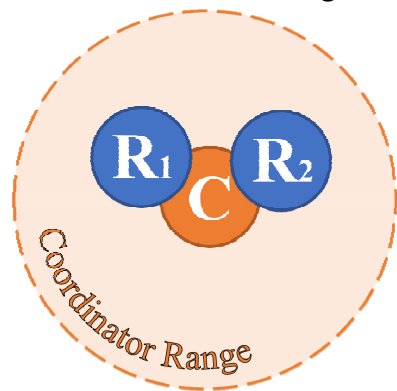

Fig. 4. Representation of Case 1.

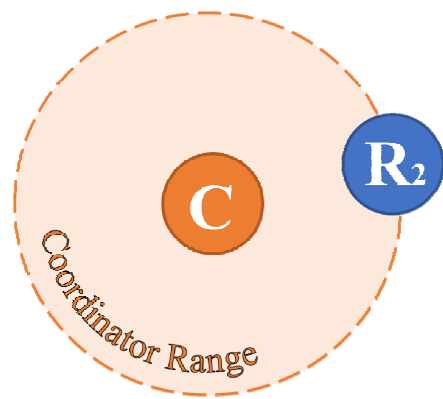

Fig. 5. Representation of Case 2.

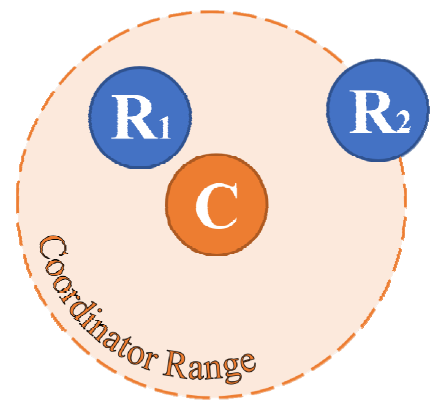

Fig. 6. Representation of Case 3.

To evaluate the ratio between the sent and received packets, the routers were programmed to send an ASCII character with a number incrementing from 0 for each packet sent, with a five second delay. Based on the serial sent by each router, through MATLAB it was possible to evaluate offline the packet reception ratio given by

$$
P R R=\frac{p r}{p s}=\frac{p r}{p r+p n r}
$$

where $p r$ is the packets received, $p s$ the packets sent, $p n r$ the packets not received.

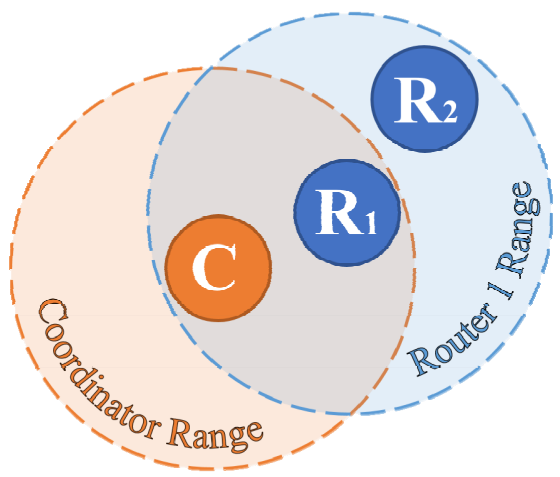

Fig. 7. Representation of Case 4 .

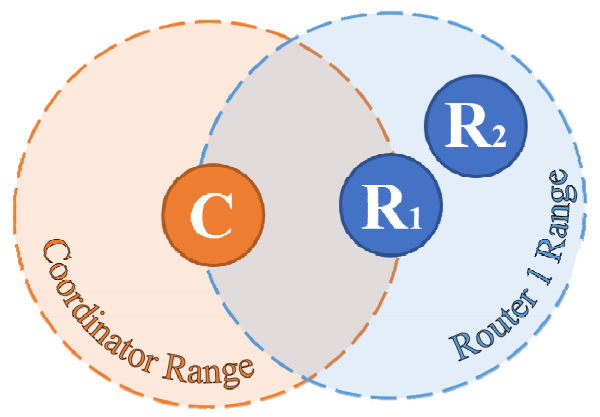

Fig. 8. Representation of Case 5 .

For a better overview, the results obtained are shown in Table I:

Case 1 Results: This case consists of having the two routers within the coordinators range. As expected the PRR was around $97 \%$ on Router 1 and $100 \%$ on Router 2 (in the experiment made). The small percentage of packets lost may be caused by the delays on the initialization process.

The time intervals between the data received for this case are shown in Fig. 9. As expected, there were no major delays in the packet reception. The expected $5 \mathrm{~s}$ delay was obtained with very low variance.

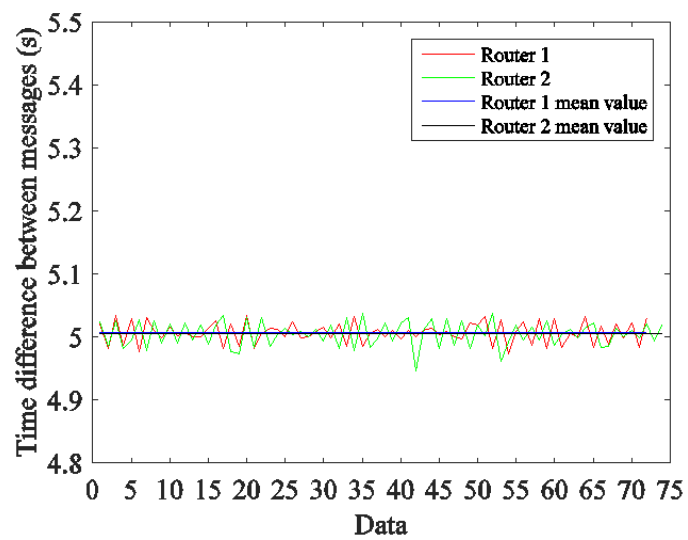

Fig. 9. Time intervals between the data received. 
TABLE I. RESULTS ACHIEVED

\begin{tabular}{|c|c|c|c|c|c|}
\hline \multirow{2}{*}{ Cases } & \multicolumn{5}{|c|}{ Data Analysis } \\
\cline { 2 - 6 } & Source & $\begin{array}{c}\text { Mean time per } \\
\text { packet (s) }\end{array}$ & $\begin{array}{c}\text { Maximum time per } \\
\text { packet (s) }\end{array}$ & $\begin{array}{c}\text { Minimum time per } \\
\text { packet (s) }\end{array}$ & $\begin{array}{c}\text { Packet reception ratio } \\
\text { (PRR) }\end{array}$ \\
\hline \multirow{3}{*}{ Case 1 } & Router 1 & 5.0068 & 5.0340 & 4.9730 & 97.33 \\
\cline { 2 - 6 } & Router 2 & 5.0047 & 5.0380 & 4.9460 & 100 \\
\hline \multirow{2}{*}{ Case 2 } & Router 1 (OFF) & NA & NA & NA & NA \\
\cline { 2 - 6 } & Router 2 & 6.1841 & 26.4100 & 0.7650 & 79.80 \\
\hline \multirow{2}{*}{ Case 3 } & Router 1 & 5.0083 & 5.1250 & 4.8730 & 96.34 \\
\cline { 2 - 6 } & Router 2 & 5.3491 & 31.1570 & 4.6940 & 92.68 \\
\hline \multirow{2}{*}{ Case 4 } & Router 1 & 5.0059 & 6.5560 & 3.4710 & 97.65 \\
\cline { 2 - 6 } & Router 2 & 5.0003 & 6.6680 & 3.3680 & 95.35 \\
\hline \multirow{2}{*}{ Case 5 } & Router 1 & 6.6024 & 20.1980 & 2.0120 & 74.75 \\
\cline { 2 - 6 } & Router 2 & 10.8373 & 127.3110 & 1.7620 & 44.33 \\
\hline
\end{tabular}

Case 2 Results: This case consists of having only one router on its range limit. The purpose of this case was to evaluate how the positioning of the router would affect the PRR. The results were not as good as in Case 1 . However, a nearly $80 \%$ PRR does not compromise the system since, in the real case, as the coordinators would be set in API mode, the system can always resend the message. In this case, the packet reception time mean was raised about $1 \mathrm{~s}$. The time intervals between the data received are shown in Fig. 10.

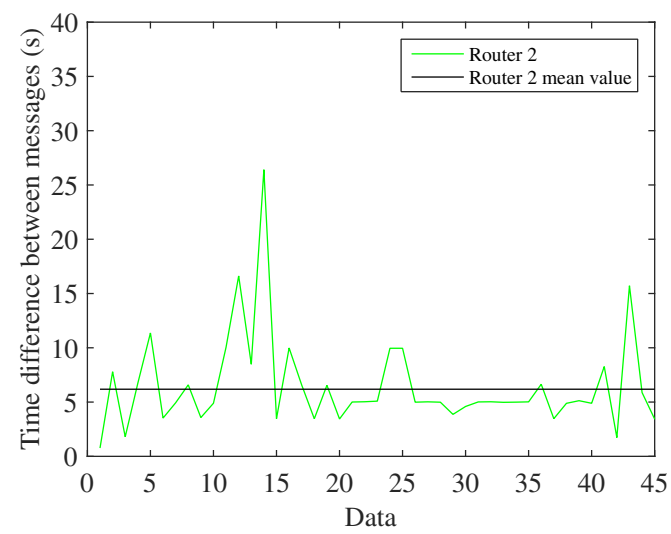

Fig. 10. Time intervals between the data.

Case 3 Results: Having a router between the limit range router and the coordinator to improve the PRR of the one in its range limit from the base. This objective was successfully achieved. Due to some issues during the start of the test, the first packet took a higher delay of time to arrive to the coordinator so the initial points shown in Fig. 11 were ignored. This way we can better see the time oscillations between packet arrivals. Nevertheless the PRR increased as expected.
Case 4 Results: In this case study, all the communications are done through the router within range since the other router is located outside the coordinators range.

The purpose is to check if the communication between the out of range router and the coordinator has been made with the help of a routing node.

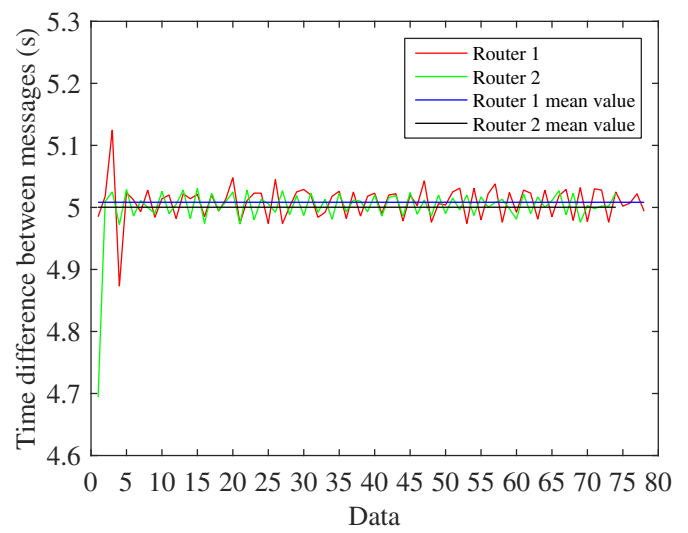

Fig. 11. Time intervals between the data received without the initial points.

The relation between the packets received by the routers is shown in Fig. 12. The PRR keeps above 90\%.

Case 5 Results: This case study corresponds to the worst case scenario. Combining the Case study 3 and the Case study 4 , i.e., one router is placed on the range limit and the other router is placed out of range of the coordinator. The influence to transmit all the communications of one router through the other one at his limit range is shown in Fig. 13.

Many packets were lost and others took a higher delay to reach the coordinator. In Table I this is the worst scenario with the lowest percentage of packet reception ratio.

These five case studies have measured the performance of the experimental setup to implement a prototype of a wireless traffic control intelligent network. 


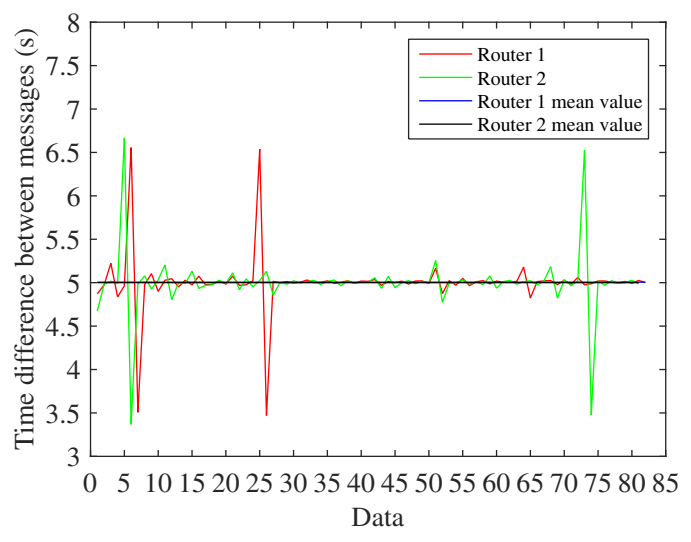

Fig. 12. Time intervals between the data received.

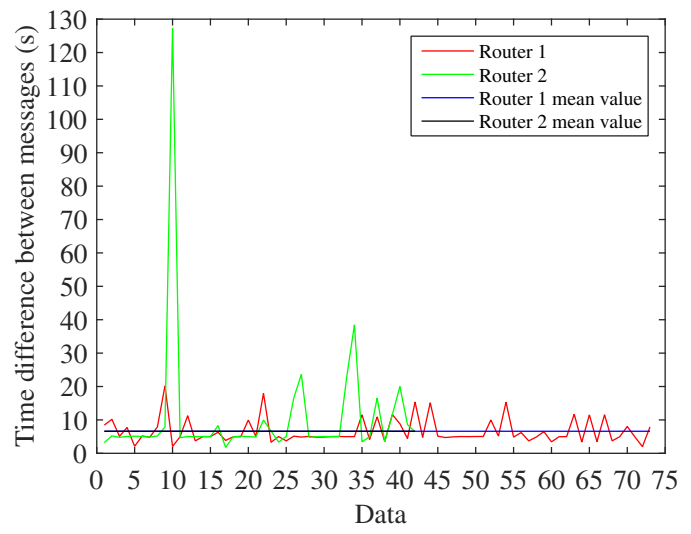

Fig. 13. Time intervals between the data received.

\section{CONClusions}

Today, ways of thinking the cities are different. The quality of life in the cities is now the main goal of its mayors and authorities. This paper contributes to find an alternative solution to outdated traffic systems, making them communicate using grid based wireless devices. The Zigbee's network properties showed good performance for the traffic system control prototype. Usually it is necessary to add, replace or remove modules from the grid for various reasons. Zigbee's uses a self-forming network so there's no need for manual reconfiguration. The possible malfunction issues on any nodes are solved due to the self-healing properties of the Zigbee network that allows the network to adjust routes from and to modules if the network changes. Regarding the communication efficiency, ZigBee provides satisfactory results, especially when in a grid. Tests were performed to limit distance situations and to push them to their extremes. Results were positive, guaranteeing that the communication between modules keeps a decent packets received ratio.

\section{ACKNOWLEDGMENTS}

This work was supported by the Portuguese Foundation for Science \& Technology, through IDMEC, under LAETA, project UID/EMS/50022/2013.

\section{REFERENCES}

[1] K.M. Yousef, J.N. Al-Karaki, and A.M. Shatnawi, "Intelligent trafic flow control system using wireless sensors networks," Journal of Information Science and Engineering, 26, 753-768, 2010.

[2] A.N. Knaian, "A wireless sensor network for smart roadbeds and intelligent transportation systems," MsC Thesis, Massachusetts Institute of Technology, USA, 2000.

[3] M.A. Vieira, "Melhoria da velocidade dos transportes públicos de superfície em Lisboa por regulação da admissão de trânsito," MsC Thesis, Instituto Superior Técnico, Portugal, 2004.

[4] http://www.jn.pt/arquivo/2004/interior/sistema-gertrude-alargado-amais-entradas-da-cidade-461054.html. [Accessed 2016-07-11].

[5] E. Franceries, and K. Liver, "Centralized traffic management system as response to the effective realization of urban traffic fluency", Archives of Transportation Telematics, vol. 4:4, pp. 4-10, 2011.

[6] Smart traffic light, https://en.wikipedia.org/wiki/Smart_traffic_light. [Accessed 2016-07-11].

[7] M.Collotta, L. Lo Bello, and G. Pau, "A novel approach for dynamic traffic lights management based on wireless sensor networks and multiple fuzzy logic controllers," Expert Systems with Applications, vol. 42, pp. 5403-5415, 2015.

[8] S. Faye; and C. Chaudet, "Characterizing the topology of an urban wireless sensor network for road traffic management," IEEE Transactions on Vehicular Technology, vol.PP(99), pp.1-7, 2015.

[9] M.A. Kafi,Y. Challal, D. Djenouri, M. Doudou, A. Bouabdallah, and N. Badache, "A study of wireless sensor networks for urban traffic monitoring: applications and architectures", Procedia Computer Science, vol. 19, pp. 617-626, 2013.

[10] R. Hussian, S. Sharma, and V. Sharma, "WSN applications : automated intelligent traffic control system using sensors," International Journal of Soft Computer and Engineering, vol. 3, pp. 77-81, 2013.

[11] Sitraffic Wimag: Wireless Magnetic Detector, Innovation in traffic detection, www.siemens.com/mobility.

[12] N.C. Batista, R. Melício, J.C.O. Matias, and J.P.S. Catalão, "Photovoltaic and wind energy systems monitoring and building/home energy management using ZigBee devices within a smart grid," Energy, vol. 49, pp. 306-315, 2013.

[13] J. Cunha, C. Cardeira, and R. Melício, "Traffic lights control prototype using wireless technologies," International Conference on Renewable Energies and Power Quality, pp. 1-6, 2016.

[14] C. Cardeira, A. W. Colombo, and R. Schoop, "Wireless solutions for automation requirements," in ATP Intern.-Automation Technology in Practice, IFAC-affiliated journal, vol. 2, September 2006, pp. 51-58.

[15] N.C. Batista, R. Melício, and V.M.F. Mendes, "Layered smart grid architecture approach and field tests by ZigBee technology," Energy Conversion and Management, vol. 88, pp. 49-59, 2014.

[16] N.C. Batista, R. Melício, J.C.O. Matias, and J.P.S. Catalão, "ZigBee standard in the creation of wireless networks for advanced metering infrastructures," 16th IEEE Mediterranean Electrotechnical Conference, pp. 220-223, 2012.

[17] N.C. Batista, R. Melício, J.C.O. Matias, and J.P.S. Catalão, "ZigBee wireless area network for home automation and energy management: field trials and installation approaches," 3rd IEEE PES Europe Conference on Innovative Smart Grid Technologies, pp. 1-5, 2012.

[18] N.C. Batista, R. Melício, J.C.O. Matias, and J.P.S. Catalão, "ZigBee devices for distributed generation management: field tests and installation approaches," 6th IET International Conference on Power Electronics, Machines and Drives, pp. 1-5, 2012.

[19] P. Koopman, and T. Chakravarty, "Cyclic redundancy code (CRC) polynomial selection for embedded networks," International Conference on Dependable Systems and Networks, pp.1-11, 2004. 